\title{
A 24-year-old woman with heavy menstrual bleeding
}

\author{
Michelle Sholzberg MDCM MSc, Jerome Teitel MD, Lisa K. Hicks MD MSc
}

Cite as: CMAJ 2017 June 5;189:E779-80. doi: 10.1503/cmaj.161469

A 24-year-old woman presents to her family physician for follow-up of bloodwork for a history of heavy menstrual bleeding from the onset of menarche. She also describes easy bruising and recurrent episodes of epistaxis. Routine coagulation studies (platelet count, activated partial thromboplastin time [aPTT] and prothrombin time [PT]) are normal. She is anemic and iron deficient, with a hemoglobin of 98 (normal range $130-170) \mathrm{g} / \mathrm{L}$ and ferritin $<10 \mu \mathrm{g} / \mathrm{L}$. Her platelet count is 390 (normal range $150-450) \times 10^{9} / \mathrm{L}$.

\section{What diagnoses should be considered in this patient?} The patient describes a lifelong history of excessive mucocutaneous bleeding suggestive of von Willebrand disease or a platelet function disorder, both of which affect about $1 \%-2 \%$ of the population. ${ }^{1,2}$ These two disorders should be at the top of the differential diagnosis. However, taking a careful medication history, even in patients with a lifelong history of bleeding, is critical to rule out exacerbating variables (e.g., concomitant use of nonsteroidal anti-inflammatory drugs or antiplatelet agents). ${ }^{3,4}$

\section{What questions are important when taking a history?}

The patient's bleeding history is an important predictor of a bleeding disorder. However, bleeding symptoms are prone to recall bias. Validated bleeding assessment questionnaires have sensitivities between $85 \%$ and $100 \%$ and are available in self-reported and clinician-administered formats. ${ }^{5-7}$ They generally include 12 to 14 questions that quantify the bleeding history and take 5 to $10 \mathrm{~min}$ utes to complete. ${ }^{6,7}$ Use of these questionnaires can obviate the need for any additional blood testing when negative (see, for example, the list of bleeding questionnaires at www.path. queensu.ca/labs/james/bq.htm). ${ }^{6-10}$

\section{What tests should be ordered?}

If the bleeding questionnaire score and family history of bleeding are negative, no further investigations are required. ${ }^{6-8,10}$ If the bleeding questionnaire score or family history of bleeding is positive, the patient requires additional evaluation. Because tests for von Willebrand disease and platelet disorders are difficult to order and interpret, patients should be referred to a hematologist for further investigation. The referral should include a description of the bleeding history (ideally with a completed bleeding questionnaire score), complete blood count and ferritin results.
Testing for hemostatic disorders requires access to a specialized laboratory, ideally located at the same site where the patient's venipuncture is completed so as to minimize the effect of pre-analytic factors on sample integrity (e.g., repeated freezing or thawing), which may distort test results. Improper sample handling is a common cause of false-positives and false-negatives. ${ }^{11}$

Reagents for measuring aPTT and PT are insensitive to minor single-factor deficiencies and are unaffected by platelet dysfunction. ${ }^{12}$ Therefore, these tests cannot be used to screen for von Willebrand disease and platelet function disorders.

\section{Where should this patient be referred?}

If the patient requires referral, she should be referred to a hematologist with expertise in bleeding disorders. This will facilitate rapid diagnosis and appropriate management. In Canada, there are 26 hemophilia treatment centres with specialized expertise in diagnosing and managing all bleeding disorders (not just hemophilia). Many of these clinics are multidisciplinary (e.g., obstetrics/gynecology, pediatrics, anesthesia, orthopedic surgery, rheumatology, physiotherapy, social work), which streamlines care and improves patient outcomes. ${ }^{13}$ The Association of Hemophilia Clinic Directors of Canada website (https://www.ahcdc. $\mathrm{ca} /$ ) has a complete list of hemophilia treatment centres (with contact information) to facilitate referral. Of note, some hemophilia treatment centres are located in rural areas. If a referral is not possible, members of the association are readily available to provide telephone or online support remotely.

\section{Case revisited}

A bleeding questionnaire (the Condensed MCMDM-1VWD) was administered and the summative score was 10 , which was suggestive of a bleeding disorder. The patient was referred to a hematologist at a hemophilia treatment centre. The hematologist ordered testing for von Willebrand disease and platelet disorders and made the diagnosis of type 1 von Willebrand disease.

The hematologist prescribed oral iron and tranexamic acid to control the patient's menses. The patient was also referred to a gynecologist affiliated with the hemophilia treatment centre, who inserted a progesterone-eluting intrauterine device under hemostatic coverage with desmopressin, to decrease the risk of its expulsion. ${ }^{14}$ 
The patient received resources containing additional information about her illness and emergency medical treatment and was connected to the Canadian Hemophilia Society for additional support and registered for continued follow-up.

\section{References}

1. Rodeghiero F, Castaman G, Dini E. Epidemiological investigation of the prevalence of von Willebrand's disease. Blood 1987;69:454-9.

2. Werner EJ, Broxon EH, Tucker EL, et al. Prevalence of von Willebrand disease in children: a multiethnic study. J Pediatr 1993;123:893-8.

3. Arachchillage DRJ, Makris M. Choosing and using non-steroidal anti-inflammatory drugs in haemophilia. Haemophilia 2016;22:179-87.

4. Serebruany VL, Malinin AI, Eisert RM, et al. Risk of bleeding complications with antiplatelet agents: meta-analysis of 338,191 patients enrolled in 50 randomized controlled trials. Am J Hematol 2004;75:40-7.

5. Rodeghiero F, Castaman G, Tosetto A, et al. The discriminant power of bleeding history for the diagnosis of type 1 von Willebrand disease: an international, multicenter study. J Thromb Haemost 2005;3:2619-26.

6. Bowman M, Mundell G, Grabell J, et al. Generation and validation of the Con- densed MCMDM-1VWD Bleeding Questionnaire for von Willebrand disease. $J$ Thromb Haemost 2008;6:2062-6.

7. Azzam HA, Goneim HR, El-Saddik AM, et al. The condensed MCMDM-1 VWD bleeding questionnaire as a predictor of bleeding disorders in women with unexplained menorrhagia. Blood Coagul Fibrinolysis 2012;23:311-5.

8. Tosetto A, Castaman G, Plug I, et al. Prospective evaluation of the clinical utility of quantitative bleeding severity assessment in patients referred for hemostatic evaluation. J Thromb Haemost 2011;9:1143-8.

9. Rydz N, James PD. The evolution and value of bleeding assessment tools. J Thromb Haemost 2012;10:2223-9.

10. Paroskie A, Gailani D, DeBaun MR, et al. A cross-sectional study of bleeding phenotype in haemophilia A carriers. Br J Haematol 2015;170:223-8.

11. Favaloro EJ, Lippi G, Adcock DM. Preanalytical and postanalytical variables: the leading causes of diagnostic error in hemostasis? Semin Thromb Hemost 2008;34:612-34.

12. Chee YL, Crawford JC, Watson HG, et al. Guidelines on the assessment of bleeding risk prior to surgery or invasive procedures. British Committee for Standards in Haematology. Br J Haematol 2008;140:496-504.

13. Pai M, Key NS, Skinner M, et al. NHF-McMaster guideline on care models for haemophilia management. Haemophilia 2016;22(Suppl 3):6-16.

14. Rimmer E, Jamieson MA, James P. Malposition and expulsion of the levonorgestrel intrauterine system among women with inherited bleeding disorders. Haemophilia 2013;19:933-8.

\section{Competing interests: None declared.}

This article has been peer reviewed.

The clinical scenario is fictional.

Affiliations: Department of Medicine (Sholzberg, Teitel, Hicks), Division of Hematology/Oncology; Department of Laboratory Medicine and Pathobiology (Sholzberg), St. Michael's Hospital, University of Toronto, Toronto, Ont.
Contributors: Michelle Sholzberg drafted the article. Michelle Sholzberg, Jerry Teitel and Lisa Hicks contributed substantially to conception and design, revised the article critically for important intellectual content, gave final approval of the version to be published and agreed to act as guarantors of the work.

Acknowledgements: The authors wish to acknowledge the contributions of Avery Longmore, Natalya O’Neill, Amy Skitch, Aziz Jiwajee, Michael Garcia and Syed Mahamad to this manuscript.

Correspondence to: Michelle Sholzberg, SholzbergM@smh.ca 